in vivo $34: 3611-3618(2020)$

doi:10.21873/invivo.12206

\title{
Plan-Optimization Method for Central-shielding Pelvic Volumetric-modulated Arc Therapy for Cervical Cancer
}

\author{
RYUTA HIRAI $^{1}$, TOMOAKI TAMAKI ${ }^{1,2}$, MITSUNOBU IGARI $^{1}$, \\ YU KUMAZAKI ${ }^{1}$, SHIN-EI NODA ${ }^{1}$ and SHINGO KATO ${ }^{1}$ \\ ${ }^{1}$ Department of Radiation Oncology, Saitama Medical University International Medical Center, Saitama, Japan; \\ ${ }^{2}$ Department of Radiation Oncology, Fukushima Medical University, Fukushima, Japan
}

\begin{abstract}
Aim: To establish a method of plan optimization in pelvic volumetric-modulated arc therapy (VMAT) for cervical cancer using the central-shielding (CS) principle. Patients and Methods: We created external beam VMAT plans for eight cases with non-bulky stage I-IIb using the CS principle based on the Japanese standard guideline. Clinical target volumes (CTVs) for whole-pelvis (WP) irradiation were created using published guidelines, and CTVs for CS irradiation were created by subtracting the uterus corpus and $4 \mathrm{~cm}$-wide regions centered at the cervix and vagina from the CTVs for WP irradiation. For plan optimization of CS irradiation, a 4-cm diameter cylindrical volume centered in the cervix and vagina was created as the volume receiving a high dose in brachytherapy, and the volume overlapping with the rectum was defined as the Ant-Rectum. Dosevolume histogram (DVH) parameters of two CS VMAT plans with and without (VMAT $T_{O P T} / V M A T_{N O}$ ) dose optimization to the Ant-Rectum were compared. Results: VMAT ${ }_{O P T}$ plans resulted in significantly lower DVH parameters of the AntRectum and rectum compared to VMAT $T_{N O}$ plans. These were comparable to the DVH parameters of three-dimensional conformal radiotherapy (3DCRT) plans. Both VMAT plans resulted in significantly better coverage of planning target volumes than did the 3DCRT plans. Conclusion: In the implementation of IMRT/VMAT as the standard treatment for cervical cancer in Japan, our optimization method may be an essential step toward fully benefitting from the CS principle.
\end{abstract}

This article is freely accessible online.

Correspondence to: Ryuta Hirai, Department of Radiation Oncology, Saitama Medical University International Medical Center, 1397-1 Hidaka, Yamane, Saitama 350-1298, Japan. Tel/Fax: +81 429844136, e-mail: rhirai@ saitama-med.ac.jp

Key Words: Cervical cancer, central-shielding, intensity-modulated radiation therapy, volumetric-modulated arc therapy, rectal toxicity.
The standard radiotherapy for uterine cervical cancer is a combination of external beam radiotherapy (EBRT) and intracavitary brachytherapy (ICBT). In Japan, standard EBRT consists of whole pelvic (WP)- and central shielding (CS)-EBRT (1). CS-EBRT is generally achieved by placing a rectangular midline block (3-4 cm width) or equivalent shielding with multileaf collimators in two opposing anterior-posterior fields, thereby reducing the radiation dose to organs at risk (OARs) such as the bladder and the rectum, while providing the necessary dose to the pelvic lymph nodes and parametria. This treatment strategy may be responsible for the relatively low incidence of late toxicities in the rectum and bladder of patients treated according to Japanese treatment protocols (2-4). Given that CS-EBRT is applied using the technique of twodimensional or three-dimensional conformal radiotherapy (3DCRT), there are several drawbacks, including inadequate shielding of OARs outside the midline block, such as the small bowel, sigmoidal colon, and pelvic bones. In addition, the shielding does not always conform to the shape of the bladder and the rectum.

Compared to 3DCRT, intensity-modulated radiotherapy (IMRT) and volumetric-modulated arc therapy (VMAT) have superior dose distributions that conform to the shape of the target volume, and as a result, yield lower acute and late radiation toxicities without compromising the treatment effects for various types of tumors. WP-IMRT/VMAT has been used increasingly to treat cervical cancer in countries which do not perform CS-EBRT and was shown to yield better dose distribution and clinical results for OARs (5-9). In order to improve standard radiotherapy for cervical cancer in Japan, we previously conducted a dose-planning study which applied IMRT/VMAT to radiotherapy for early-stage non-bulky cervical cancer cases. For CS-EBRT in that study, we re-examined the principle of CS and defined the specific clinical target volume (CTV) of CS-EBRT, a crucial step in the treatment planning of IMRT/VMAT (10). Nonetheless, the original purpose of CS-EBRT, which is to specifically shield the bladder and the rectum, must be maintained in CS-VMAT. 

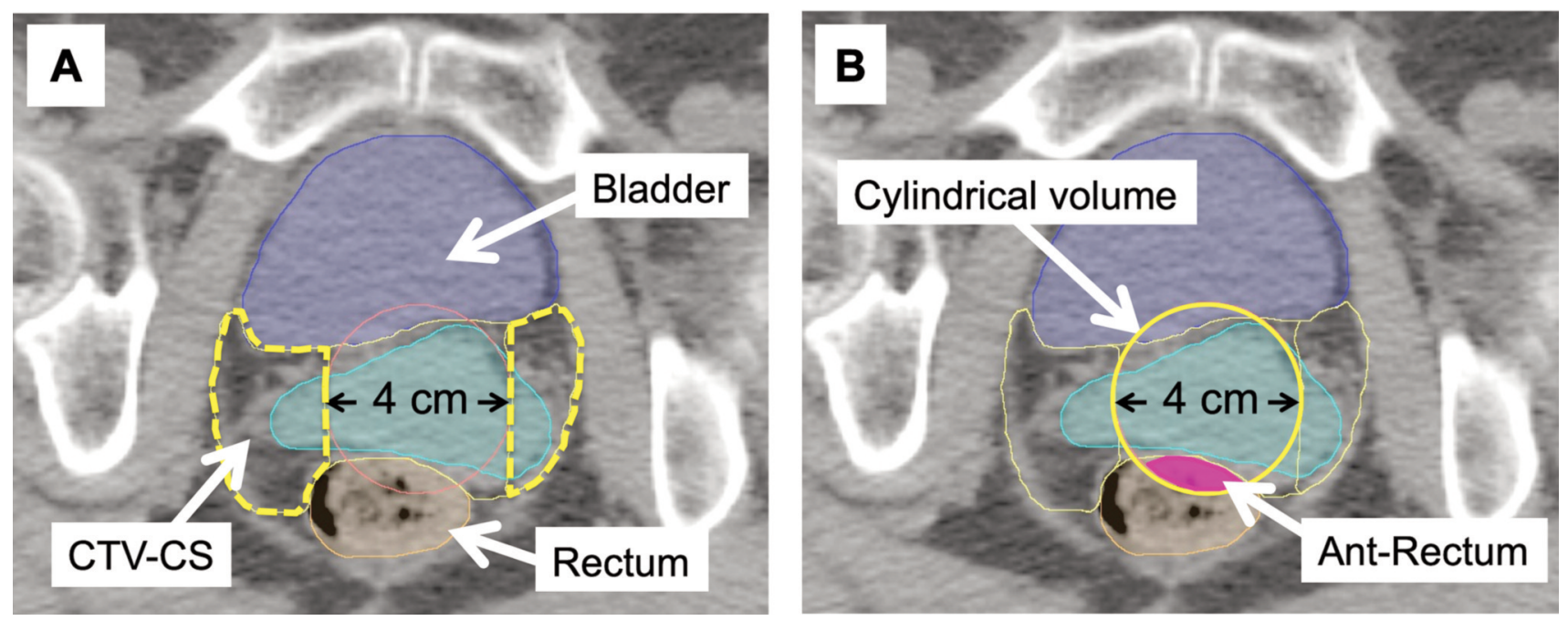

Figure 1. Schematics of the definition of the clinical target volume with central-shielding external beam radiotherapy (CTV-CS) (A) and the AntRectum $(B)$. The cylindrical volume was positioned in the cervix and vaginal canal.

The principle of CS can easily be visualized and is conceptually clear in 3CDRT, given the presence of midline block/shielding. However, it may be unclear and easily overlooked in the planning of IMRT/VMAT because optimizing the doses simply for the planning target volume (PTV) and the overall volume of OARs may not be sufficient to achieve the original purpose. It is essential to identify specific regions with high risk of radiation toxicities, i.e. regions which receive high doses from ICBT (ICBT hotspot). In radiotherapy for cervical cancer, the anterior rectal wall is one of the most critical areas of OARs because it is in close proximity to the cervical tumor and susceptible to receiving a high radiation dose from the combination of EBRT and ICBT $(11,12)$. The reported dose constraint for the anterior rectal wall is lower than that for the posterior wall of the bladder $(13,14)$, and reducing the radiation dose to the anterior rectal wall is important in minimizing the risk of late rectal toxicities and overall toxicities $(15,16)$. In fact, the concept of optimizing the dose to ICBT hotspots in CS-VMAT planning is a unique concept for the following two reasons. Firstly, unlike other types of cancer, cervical cancer requires not only EBRT but a combination of EBRT and ICBT. Secondly, as opposed to CS-VMAT, the PTV of WP-VMAT may frequently include the anterior rectal wall and therefore provides no opportunity to lower the dose to the ICBT hotspot.

In the present study, we introduced a dose optimization method which entails identifying the ICBT hotspot in the anterior rectal wall. We analyzed dose distributions and dosevolume histograms (DVHs) of CS-VMAT and evaluated treatment plans which were optimized only for the overall OARs or additionally for the identified ICBT hotspot. Our results showed the effectiveness of the optimization method, which minimize the dose to the ICBT hotspot in the anterior rectal wall, and highlights the importance of the method when implementing CS-VMAT.

\section{Patients and Methods}

Delineation of target volumes and OARs. This study was approved by the Institutional Review Board of our Institute. Computed tomographic image data of eight patients with cervical cancer who underwent definitive radiotherapy at our hospital from January 2015 to August 2015 were analyzed in the study. All patients had stage IIIB disease with a maximum tumor size $<4 \mathrm{~cm}$. Definitions of the CTVs for non-bulky cervical cancer cases were defined and described in a previous report on the application of WP-VMAT and CS-VMAT (10). Briefly, the CTVs for primary disease of the uterine cervix (CTV-PD) and pelvic lymph nodes (CTV-LN) were delineated according to published guidelines $(17,18)$. CTV-WP was defined as the sum of the CTV-PD and CTV-LN. In order to create the CTV-CS, the uterus corpus and a $4-\mathrm{cm}$ wide region centered at the cervical canal and the center of vagina, but not at the center of the pelvic bony structure, were subtracted from the CTV-WP (Figure 1A). The outer contours of the bladder, rectum, bowel bag (small and large intestines), and the bilateral femoral head were delineated as OARs. In addition, a 4-cm diameter cylindrical volume centered in the cervix and vaginal canal was created and represented the volume irradiated with a high dose in ICBT. The overlapping region between the rectum and the 4-cm diameter cylindrical volume was defined as the dummy structure, Ant-Rectum, which represented the high-risk region of radiation rectal toxicity caused by high dose irradiation (Figure 1B). The Ant-Rectum was created for the optimization process to minimize the anterior rectal dose in CS-VMAT.

Planning. In this planning study, we postulated that all patients were treated with $20 \mathrm{~Gy} / 10$ fractions of WP-EBRT and $30 \mathrm{~Gy} / 15$ fractions of CS-EBRT according to Japanese guidelines (2-4). For the treatment planning of CS-EBRT, a PTV margin of $5 \mathrm{~mm}$ was 
A

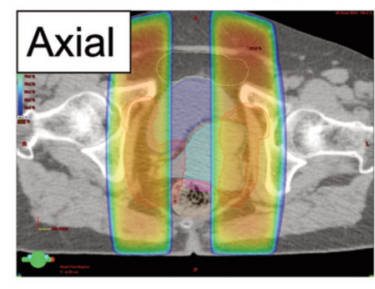

B

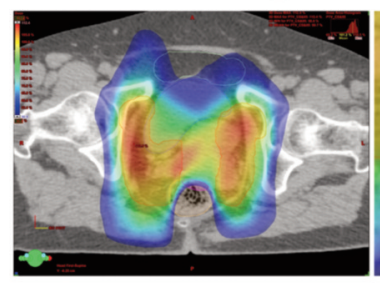

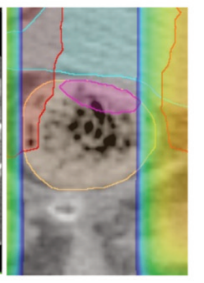
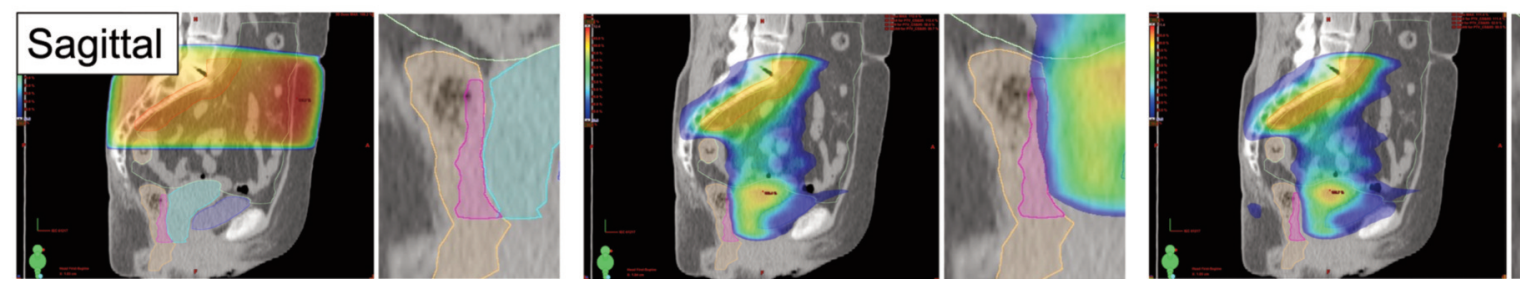

C
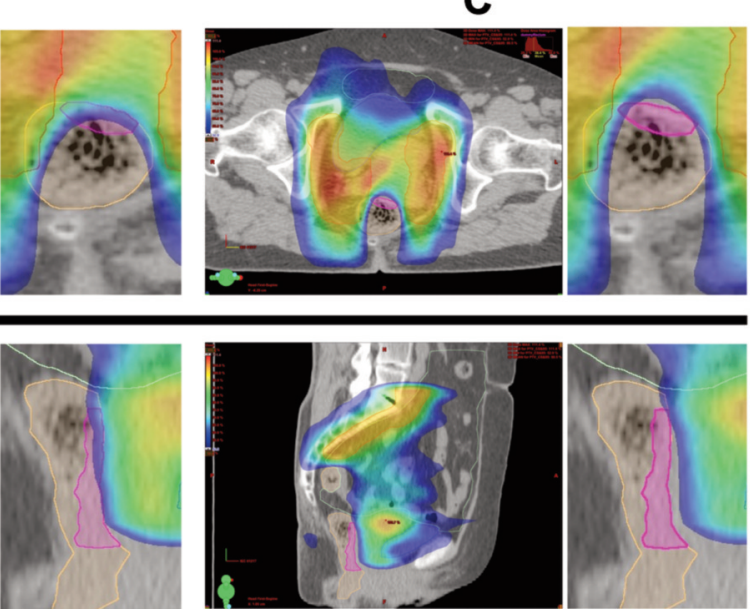

Figure 2. Dose distributions of three-dimensional conformal radiotherapy (A), volumetric-modulated arc therapy (VMAT) without dose optimization for the Ant-Rectum (VMAT $T_{\mathrm{NO}}$ ) (B), and VMAT with dose optimization for the Ant-Rectum (VMAT $\left.T_{\mathrm{OPT}}\right)(C)$. The upper panel shows axial views and the lower shows sagittal views. Dose range of $50 \%$ or more of the prescribed dose is indicated by color wash.

added to the CTV-CS, and the following three treatment plans were evaluated: 3DCRT, VMAT with dose optimization only for the OARs ( VMAT $_{\mathrm{NO}}$ ), and VMAT with dose optimization for the AntRectum in addition to the OARs (VMAT $\mathrm{OPT}_{\mathrm{OPT}}$ ). For 3DCRT, the anterior and posterior parallel opposed portals were created, and a $3-\mathrm{cm}$ wide CS was placed at the center of the bony pelvis. The height of the CS was adjusted such that it did not compromise the presacral lymph node region. A total dose of $30 \mathrm{~Gy} / 15$ fractions was delivered using $10 \mathrm{MV}$ X-rays. For VMAT planning, a double-arc VMAT plan using $10 \mathrm{MV}$ x-ray beams was created for the PTV-CS. The dose prescription was based on PTV-CS $D_{50 \%}$ values with the planning aims as follows: PTV-CS $D_{95 \%}>92 \%$ and PTV-CS $D_{2 \%}<110 \%$. For $\mathrm{VMAT}_{\mathrm{NO}}$, planning aims for OARs were set as follows: for the rectum, volume receiving $24 \mathrm{~Gy}\left(V_{24 \mathrm{~Gy}}\right)<85 \%$, volume receiving $30 \mathrm{~Gy}\left(V_{30} \mathrm{~Gy}\right)<40 \%$, the maximum dose $\left(D_{\max }\right)<110 \%$; for the bladder, $D_{\max }<110 \%$; for the bowel bag, $V_{24}$ Gy $<40 \%$; for the femoral heads, $V_{18 \mathrm{~Gy}}<40 \%$; and for the whole body, $D_{\max }<115 \%$. For $\mathrm{VMAT}_{\mathrm{OPT}}$, in addition to meeting the planning aims of $\mathrm{VMAT}_{\mathrm{NO}}$, the plans were optimized to reduce the dose to the Ant-Rectum to the maximum extent while maintaining the required coverage of the PTV-CS $\left(D_{95 \%}>92 \%\right)$. All calculations were performed using the treatment planning system Eclipse version 11 with Analytical Anisotropic Algorithm (AAA, ver. 11.0.31; (Varian Medical Systems, Inc., Palo Alto, CA, USA). To evaluate the treatment plans, $D_{95 \%}, D_{98 \%}$, and $D_{2 \%}$ of the PTV-CS, $V_{60 \%}$, $V_{80 \%}, D_{\text {max }}$, and $D_{2} \mathrm{~cm}^{3}$ of the rectum, bladder, and the bowel bag were analyzed for $3 \mathrm{DCRT}, \mathrm{VMAT}_{\mathrm{NO}}$, and $\mathrm{VMAT}_{\mathrm{OPT}}$ plans. In order to assess the dose to the anterior rectal wall, we also analyzed $V_{60 \%}$, $V_{80 \%}, D_{\max }$, and $D_{2 \mathrm{~cm}^{3}}$ of the Ant-Rectum. Parameters for each plan were compared using the non-parametric Wilcoxon signed-rank test. $p$-Values less than 0.05 were considered statistically significant.

\section{Results}

The Ant-Rectum was contoured successfully in all studied cases, and the average volume \pm SD of the Ant-Rectum was
$6.5 \pm 3.3 \mathrm{~cm}^{3}$. Overlap of the PTV-CS and the Ant-Rectum was minimal $\left(0.2 \pm 0.2 \mathrm{~cm}^{3}\right)$.

Dose distributions of the 3DCRT, VMAT $\mathrm{NO}_{\mathrm{NO}}$, and VMAT $_{\text {OPT }}$ plans of a case are shown in Figure 2, and representative DVHs of the PTV-CS, rectum, Ant-Rectum, bladder, and the bowel bag are shown in Figure 3. Table I summarizes the DVH parameters of the eight cases. Most of the Ant-Rectum was directly shielded by the midline block in 3DCRT plans. DVH curves revealed that the dose to the Ant-Rectum was lower in VMAT $_{\text {OPT }}$ plans than that in VMAT $_{\text {NO }}$ plans. When comparing DVH parameters of the Ant-Rectum, all parameters of VMAT $_{\text {OPT }}$ plans were significantly lower than those of $\mathrm{VMAT}_{\mathrm{NO}}$ plans. VMAT $\mathrm{OPT}_{\mathrm{O}}$ plans showed almost equivalent $V_{60 \%}, V_{80 \%}$, and $D_{\max }$ to 3DCRT plans. Regarding DVH parameters of the rectum, VMAT $_{\text {OPT }}$ plans showed significantly lower $V_{60 \%}, V_{80 \%}$, and $D_{2} \mathrm{~cm}^{3}$ than those of $\mathrm{VMAT}_{\mathrm{NO}}$ plans. VMAT $\mathrm{NO}$ and VMAT $_{\text {OPT }}$ plans showed significantly lower $V_{80 \%}$ than that of 3DCRT plans, although $V_{60 \%}, D_{\max }$, and $D_{2} \mathrm{~cm}^{3} \mathrm{did}$ not differ significantly between $3 \mathrm{DCRT}$ and $\mathrm{VMAT}_{\mathrm{NO} / \mathrm{OPT}}$ plans. For the bowel bag, VMAT $\mathrm{NO}_{\mathrm{NO}}$ and $\mathrm{VMAT}_{\mathrm{OPT}}$ plans showed comparable DVH parameters $\left(D_{\max }\right.$ of the bowel bag was

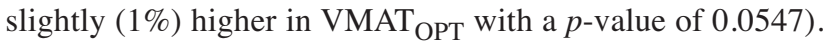
Compared to 3DCRT plans, both $\mathrm{VMAT}_{\mathrm{NO}}$ and $\mathrm{VMAT}_{\mathrm{OPT}}$ plans had lower $V_{60 \%}$ and $V_{80 \%}$ and higher $D_{\max }$. For the bladder, $\mathrm{VMAT}_{\mathrm{NO}}$ and $\mathrm{VMAT}_{\mathrm{OPT}}$ plans had comparable DVH parameters $\left(V_{60 \%}\right.$ of the bladder was slightly $(2.2 \%)$ higher in $\mathrm{VMAT}_{\mathrm{OPT}}$ with a $\left.p=0.0547\right)$. Compared to 3DCRT plans, both VMAT $_{\text {NO }}$ and VMAT $_{\text {OPT }}$ plans had lower $V_{80 \%}$ and higher $V_{60 \%}$ and $D_{\max }$.

In Figure 2A, the medial portion of the PTV-CS which had midline shielding did not receive a high dose in the 

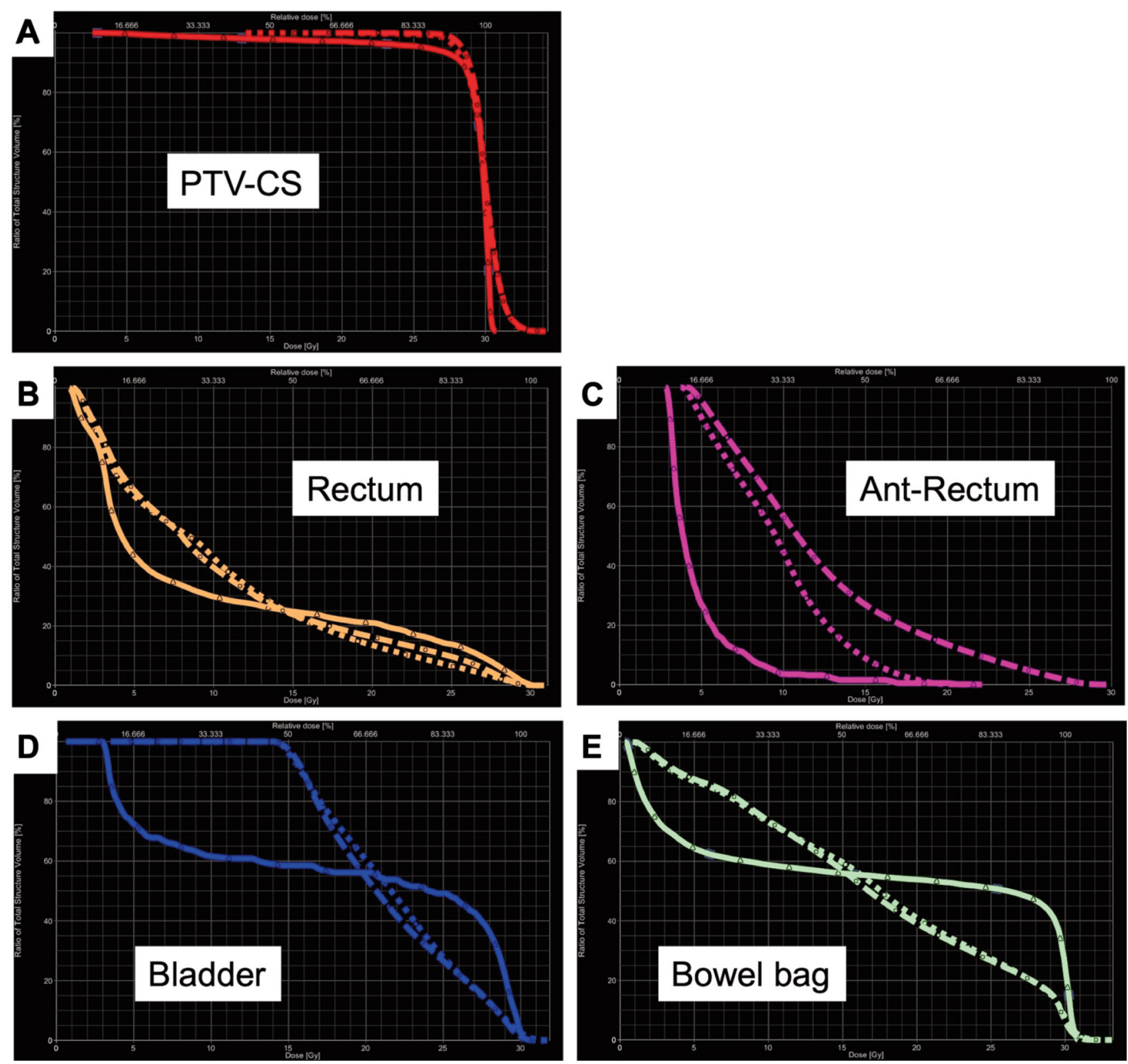

Figure 3. Representative dose-volume histograms (DVHs) of the planning target volume with central-shielding (PTV-CS) (A), rectum (B), Ant-Rectum $(C)$, bladder $(D)$, and bowel bag (E) comparing three-dimensional conformal radiotherapy (3DCRT) and volumetric-modulated arc therapy (VMAT) plans in clinical cases. Solid line: DVH curve of 3DCRT plan; dashed line: DVH curve of VMAT plan without dose optimization for the Ant-Rectum $\left(V M A T_{\mathrm{NO}}\right)$; dotted line: DVH curve of VMAT plan with dose optimization for the Ant-Rectum (VMAT $\left.T_{\mathrm{OPT}}\right)$.

3DCRT plan. In contrast, this portion was covered with a high dose in both $\mathrm{VMAT}_{\mathrm{NO}}$ and $\mathrm{VMAT}_{\mathrm{OPT}}$ plans (Figure $2 \mathrm{~B}$ and $\mathrm{C}$ ). DVH curves also revealed the higher dose coverage of the PTV-CS in both VMAT $_{\mathrm{NO}}$ and $\mathrm{VMAT}_{\mathrm{OPT}}$ plans compared to that in the 3DCRT plan (Figure 3A). DVH parameters also showed improved coverage of the PTV-CS in both $\mathrm{VMAT}_{\mathrm{OPT}}$ and $\mathrm{VMAT}_{\mathrm{NO}}$ plans compared to the 3DCRT plan, as reflected in the significantly higher $D_{95 \%}$ and $D_{98 \%}$ of the PTV-CS. When comparing the two VMAT plans, the VMAT OPT $_{\text {OP }}$ plan had lower $D_{95 \%}$ and $D_{98 \%}$ than those of the VMAT $_{\text {NO }}$ plan.

\section{Discussion}

In radiotherapy for cervical cancer, late rectal toxicities, such as a radiation proctitis, rectal ulcer, and rectovaginal fistula, 
Table I. Comparison of parameters of the planning target volume with central-shielding (PTV-CS) and the Ant-Rectum between three-dimensional conformal radiotherapy (3DCRT) and volumetric-modulated arc therapy (VMAT) plans.

\begin{tabular}{|c|c|c|c|c|c|c|c|}
\hline \multirow{2}{*}{$\begin{array}{l}\text { Volume of interest } \\
\text { (average } \pm \text { SD) }\end{array}$} & \multirow[t]{2}{*}{ Parameter } & \multirow[t]{2}{*}{ 3DCRT } & \multirow[t]{2}{*}{$\mathrm{VMAT}_{\mathrm{NO}}$} & \multirow[t]{2}{*}{$\mathrm{VMAT}_{\mathrm{OPT}}$} & \multicolumn{3}{|c|}{$p$-Values } \\
\hline & & & & & $\begin{array}{l}\text { 3DCRT } v s . \\
\text { VMATNO }\end{array}$ & $\begin{array}{l}\text { 3DCRT } v s . \\
\text { VMATOPT }\end{array}$ & $\begin{array}{l}\text { VMATNO } v s \\
\text { VMATOPT }\end{array}$ \\
\hline PTV-CS & $D_{95 \%}$ & $84.4 \pm 9.8$ & $93.4 \pm 0.7$ & $92.8 \pm 0.8$ & 0.0391 & 0.0547 & 0.0234 \\
\hline \multirow[t]{2}{*}{$\left(760.7 \pm 71.4 \mathrm{~cm}^{3}\right)$} & $D_{98 \%}$ & $45.5 \pm 23.9$ & $91.1 \pm 1.0$ & $90.1 \pm 1.3$ & 0.0078 & 0.0078 & 0.0234 \\
\hline & $D_{2 \%}$ & $101.9 \pm 0.8$ & $106.3 \pm 0.7$ & $106.3 \pm 0.5$ & 0.0078 & 0.0078 & 0.8438 \\
\hline Rectum & $V_{60 \%}$ & $21.4 \pm 8.8$ & $16.7 \pm 3.8$ & $15.2 \pm 4.6$ & 0.1953 & 0.1484 & 0.0391 \\
\hline \multirow[t]{3}{*}{$\left(58.4 \pm 23.2 \mathrm{~cm}^{3}\right)$} & $V_{80 \%}$ & $15.8 \pm 7.9$ & $7.7 \pm 3.3$ & $6.5 \pm 3.0$ & 0.0156 & 0.0156 & 0.0234 \\
\hline & $D_{\max }$ & $99.1 \pm 2.6$ & $100.4 \pm 3.1$ & $99.2 \pm 2.7$ & 0.1953 & 0.9453 & 0.0781 \\
\hline & $D_{2} \mathrm{~cm}^{3}$ & $89.8 \pm 18.3$ & $87.3 \pm 6.9$ & $85.3 \pm 6.9$ & 0.1953 & 0.1953 & 0.0078 \\
\hline Ant-Rectum & $V_{60 \%}$ & $1.5 \pm 2.0$ & $17.1 \pm 8.3$ & $0.9 \pm 0.7$ & 0.0078 & $>0.999$ & 0.0078 \\
\hline \multirow[t]{3}{*}{$\left(6.5 \pm 3.3 \mathrm{~cm}^{3}\right)$} & $V_{80 \%}$ & $0.7 \pm 1.2$ & $3.6 \pm 3.4$ & $0.0 \pm 0.0$ & 0.0781 & 0.5000 & 0.0156 \\
\hline & $D_{\max }$ & $65.0 \pm 26.8$ & $91.1 \pm 6.2$ & $71.4 \pm 6.3$ & 0.0234 & 0.5469 & 0.0078 \\
\hline & $D_{2} \mathrm{~cm}^{3}$ & $15.8 \pm 5.1$ & $46.2 \pm 18.5$ & $33.0 \pm 9.5$ & 0.0078 & 0.0078 & 0.0156 \\
\hline Bowel bag & $V_{60 \%}$ & $58.7 \pm 3.9$ & $46.9 \pm 6.0$ & $47.9 \pm 5.8$ & 0.0078 & 0.0078 & 0.1484 \\
\hline \multirow[t]{3}{*}{$\left(1457.0 \pm 559.4 \mathrm{~cm}^{3}\right)$} & $V_{80 \%}$ & $55.6 \pm 3.8$ & $28.7 \pm 4.1$ & $29.0 \pm 4.3$ & 0.0078 & 0.0078 & 0.7109 \\
\hline & $D_{\max }$ & $103.8 \pm 1.9$ & $107.3 \pm 1.4$ & $108.3 \pm 2.1$ & 0.0156 & 0.0156 & 0.0547 \\
\hline & $D_{2} \mathrm{~cm}^{3}$ & $103.4 \pm 1.8$ & $104.3 \pm 1.2$ & $104.6 \pm 1.2$ & 0.3828 & 0.1484 & 0.1406 \\
\hline Bladder & $V_{60 \%}$ & $49.2 \pm 9.4$ & $68.2 \pm 16.7$ & $70.4 \pm 14.6$ & 0.0156 & 0.0078 & 0.0547 \\
\hline \multirow[t]{3}{*}{$\left(83.6 \pm 44.1 \mathrm{~cm}^{3}\right)$} & $V_{80 \%}$ & $42.8 \pm 10.2$ & $33.6 \pm 9.5$ & $34.4 \pm 9.1$ & 0.1953 & 0.1953 & 0.1953 \\
\hline & $D_{\max }$ & $100.8 \pm 2.0$ & $104.9 \pm 2.1$ & $105.0 \pm 1.4$ & 0.0078 & 0.0156 & 0.5469 \\
\hline & $D_{2} \mathrm{~cm}^{3}$ & $99.5 \pm 2.5$ & $98.5 \pm 2.8$ & $98.3 \pm 3.2$ & 0.25 & 0.1953 & 0.6172 \\
\hline
\end{tabular}

VMAT $_{\mathrm{NO}}$ : VMAT plan without dose optimization for the Ant-Rectum; VMAT $\mathrm{OPT}_{\mathrm{V}}$ VMAT plan with dose optimization for the Ant-Rectum. Parameters are given in percentages (\%, for both volume and dose). Statistically significant $p$-values are shown in bold.

are some of the most common and critical radiation complications. The incidence and severity of rectal toxicity depend on the dose received by the rectum, and it is often recommended that the total rectal dose $\left(D_{2} \mathrm{~cm}^{3}\right.$; minimum dose delivered to $2 \mathrm{~cm}^{3}$ of the most irradiated tissue volume) should be less than 70-75 $\operatorname{Gy}_{\mathrm{EQD} 2}(13,14)$. Therefore, minimizing the dose to the rectum, particularly to the anterior rectal wall, is of great importance.

For cervical cancer, use of the IMRT/VMAT technique in WP-EBRT has been shown to reduce toxicities in OARs such as the bowel and bone marrow (5-9). However, the CTV-PD extends very close or adjacent to the rectum by definition (17), resulting in a PTV which includes the anterior rectal wall in most cases. Therefore, it is often theoretically impossible to significantly reduce the dose to the anterior rectal wall in WP-EBRT. Consequently, the combination of WP-IMRT/VMAT and ICBT may deliver an unacceptably high dose to the anterior rectal wall. If CSEBRT is applied, a high dose to the anterior rectal wall can possibly be avoided. Defining the CTV-CS by excluding the central portion of the cervix (or cercal cancer) and the vagina from the CTV (10) leads to a PTV (PTV-CS) which does not overlap with ICBT hotspots. Our present study demonstrates this advantage of CS-EBRT, since the overlap between the PTV-CS and the Ant-Rectum was minimal.
In IMRT treatment planning, allocation of dummy structures is occasionally used to lower the radiation dose to OARs. Leicher et al. introduced a method to allocate a dummy structure in the anterior part of the abdomen to lower the dose to the small bowel in IMRT planning for rectal and anal cancer (19). Neil introduced a method to improve the target conformity and lower OAR doses by setting two kinds of dummy structures around the target in IMRT planning for prostate cancer (20). These methods are routinely utilized in the treatment planning of these cancers and are essential for producing optimal plans. The main objective of CS-EBRT is to minimize the dose to the anterior rectal wall, more precisely, to minimize the radiation dose to the area which is at a high risk of developing late complications. Therefore, it is logical to define this volume as a dummy structure to be used for plan optimization. In this study, we created the dummy structure, the Ant-Rectum, which corresponded to the volume of the rectum expected to receive a high dose in ICBT (ICBT hotspot), and optimized the dose to this volume. To the best of our knowledge, introduction of the concept of 'expected high-dose volume in brachytherapy' or 'high-risk volume' into EBRT plan optimization has not been reported previously.

The present study showed that identification of the AntRectum as the dummy structure for IMRT/VMAT 
optimization is an essential step in the treatment planning of CS-VMAT, and that optimization only for overall OAR volumes (e.g. the rectum) may not be sufficient. $\mathrm{VMAT}_{\mathrm{OPT}}$ plans showed more favorable DVH parameters of the AntRectum and the whole rectum than VMAT $_{\text {NO }}$ plans, which were comparable to those of 3DCRT plans at least in the high dose range. As for the bowel bag and bladder, $\mathrm{VMAT}_{\mathrm{NO}}$ and $\mathrm{VMAT}_{\mathrm{OPT}}$ plans showed comparable DVH parameters. Based on these results, we considered the plan-optimization method to be an effective way to minimize the dose to the anterior rectal wall in CS-VMAT while maintaining a good dose distribution to other OARs.

Both VMAT plans had significantly higher $D_{98 \%}$ and $D_{2 \%}$ of the PTV-CS compared to 3DCRT plans. $D_{95 \%}$ and $D_{98 \%}$ of the PTV-CS were lower in VMAT $_{\text {OPT }}$ plans than those in VMAT $_{\text {NO }}$ plans. However, the magnitude of the decrease was approximately $1 \%(0.3 \mathrm{~Gy})$, and thus its clinical impact was unlikely to be critical. In CS-VMAT plans, the 4-cm wide volume within the cervix and vagina is excluded from the CTV-PD, and the radiation dose to the whole CTV-PD is logically reduced by the proportion of the CS-VMAT in EBRT. However, this volume can be irradiated with very high doses in ICBT, which compensates for dose reduction in CS-EBRT $(21,22)$.

Macdonald et al. (23) and Kidd et al. (24) defined the target for conventional split-field 3DCRT irradiation using the step-wedge technique in order to delineate the primary disease of cervical cancer with reference to positronemission tomography images with ${ }^{18} \mathrm{~F}$-fluorodeoxyglucose and excluded it from the target of WP-EBRT. This resulted in a transition to IMRT with the pseudo-step-wedge technique and a reduction of doses to and toxicities of OARs (23-25). Their treatment concept, pseudo-step-wedge intensity modulation (PSWIM), appears to be similar to VMAT $_{\mathrm{NO}}$. Cheng et al. also reported that the simultaneous integrated boost technique using IMRT/VMAT to boost the parametrium dosimetrically produced better target coverage but resulted in slightly higher doses to OARs (26). In our study, however, it was possible to reduce the dose to the anterior rectal wall to the maximum extent while maintaining the dose coverage to the PTV by creating the Ant-Rectum and optimizing the dose to the anterior rectal wall. Thus, the dose to the anterior rectal wall in our VMAT $_{\text {OPT }}$ plan might be lower than that reported by them. Nonetheless, our plan-optimization method might also be applicable to the PSWIM technique proposed by Macdonald et al. and Kidd et al., the simultaneous integrated boost technique proposed by Cheng et al., and other IMRT plans for cervical cancer.

Another major OAR in the radiotherapy for cervical cancer is the bladder. Specifically, the posterior wall of the bladder represents a potential OAR which may provide an opportunity for further plan optimization. However, the dose constraint for the bladder is generally higher than that for the rectum, and we concentrated on use of the Ant-Rectum to improve our plans. Moreover, in a previous study that analyzed the composite dose distribution of WP and CSEBRT and ICBT, the benefit of dose reduction from CS was greater in the rectum than in the bladder, and the bladder received a relatively large dose contribution from the high dose region at the outside of CS (21). Further studies aimed at improving plans by additionally optimizing for potential hotspots in the bladder may be warranted.

This study has several limitations. First, this was a radiotherapy dosimetric study which evaluated the feasibility of CS-VMAT with the plan-optimization method. Therefore, the sample size was small, and there may have been patient selection bias. All patients had tumors $<4 \mathrm{~cm}$, therefore a 4$\mathrm{cm}$ diameter cylindrical volume centered in the cervix and vaginal canal was created to represent the volume irradiated with high dose in standard ICBT. This volume may need to be adjusted, particularly when the dose of ICBT is escalated in order to treat bulky cervical cancer. Further studies to confirm the effectiveness of the optimization method are needed using a large sample population with various stages of cervical cancer. Secondly, inter- and intra-fractional organ positional uncertainties are major issues in EBRT for cervical cancer, suggesting the need to further examine optimum internal target volume (ITV) margins and appropriate conditions for adaptive image-guided radiotherapy. Thirdly, the high dose region in ICBT was simplified to the $4-\mathrm{cm}$ diameter cylindrical volume. However, the high-dose region in ICBT may change according to applicator position and treatment planning. Despite these limitations, we consider the present study to be important with regard to enabling optimal treatment planning, because CS-IMRT/VMAT without using this method may result in high-dose irradiation to the anterior rectal wall.

In summary, the plan-optimization method introduced in the present study resulted in significantly improved dose distributions of the CS-VMAT plan by identifying ICBT hotspots in the anterior rectal wall. This optimization method may be an essential step in the implementation of IMRT/VMAT which applies the principle of CS in radiotherapy for cervical cancer.

\section{Conflicts of Interest}

The Authors declare that there are no conflicts of interest.

\section{Author's Contributions}

All Authors contributed to the study conception and design. Material preparation, data collection and analysis were performed by RH, TT, and MI. The first draft of the article was written by $\mathrm{RH}$ and all Authors commented on previous versions of the article. All Authors read and approved the final article. 


\section{Acknowledgements}

This study was approved by the Institutional Review Board of Saitama Medical University International Medical Center (Authorization number: 15-150). This was a retrospective study, and thus the requirement for written informed consent was waived. This work was partially supported by MEXT KAKENHI $16 \mathrm{H} 05388$.

\section{References}

1 Japan Society of Gynecologic Oncology (ed.): Guidelines for Treatment of Uterine Cervical Cancer, The $3^{\text {rd }}$ Edition. Tokyo: Kanehara Shuppan, 2017.

2 Nakano T, Kato S, Ohno T, Tsujii H, Sato S, Fukuhisa K and Arai T: Long-term results of high-dose rate intracavitary brachytherapy for squamous cell carcinoma of the uterine cervix. Cancer 103: 92-101, 2005. PMID: 15540233. DOI: 10.1002/ cncr.20734

3 Toita T, Kato S, Niibe Y, Ohno T, Kazumoto T, Kodaira T, Kataoka M, Shikama N, Kenjo M, Tokumaru S, Yamauchi C, Suzuki O, Sakurai H, Numasaki H, Teshima T, Oguchi M, Kagami Y, Nakano T, Hiraoka M and Mitsuhashi N: Prospective multi-institutional study of definitive radiotherapy with high-doserate intracavitary brachytherapy in patients with nonbulky $(<4-\mathrm{cm})$ stage I and II uterine cervical cancer (JAROG0401/JROSG04-2). Int J Radiat Oncol Biol Phys 82: e49-56, 2012. PMID: 21470794. DOI: $10.1016 /$ j.ijrobp.2011.01.022

4 Toita T, Kitagawa R, Hamano T, Umayahara K, Hirashima Y, Aoki Y, Oguchi M, Mikami M and Takizawa K: Phase II study of concurrent chemoradiotherapy with high-dose-rate intracavitary brachytherapy in patients with locally advanced uterine cervical cancer: Efficacy and toxicity of a low cumulative radiation dose schedule. Gynecol oncol 126: 211216, 2012. PMID: 22555110. DOI: 10.1016/j.ygyno.2012.04.036

5 Roeske JC, Lujan A, Rotmensch J, Waggoner SE, Yamada D and Mundt AJ: Intensity-modulated whole pelvic radiation therapy in patients with gynecologic malignancies. Int J Radiat Oncol Biol Phys 48: 1613-1621, 2000. PMID: 11121668. DOI: 10.1016/s0360-3016(00)00771-9

6 Mundt AJ, Mell LK and Roeske JC: Preliminary analysis of chronic gastrointestinal toxicity in gynecology patients treated with intensity-modulated whole pelvic radiation therapy. Int J Radiat Oncol Biol Phys 56: 1354-1360, 2003. PMID: 12873680. DOI: $10.1016 / \mathrm{s} 0360-3016(03) 00325-0$

7 Hasselle MD, Rose BS, Kochanski JD, Nath SK, Bafana R, Yashar CM, Hasan Y, Roeske JC, Mundt AJ and Mell LK: Clinical outcomes of intensity-modulated pelvicradiation therapy for carcinoma of the cervix. Int J Radiat Oncol Biol Phys 80: 1436-1445, 2011. PMID: 20708346. DOI: 10.1016/j.ijrobp. 2010.04.041

8 Klopp AH, Yeung AR, Deshmukh S, Gil KM, Wenzel L, Westin SN, Gifford K, Gaffney DK, Small W Jr, Thompson S, Doncals DE, Cantuaria GHC, Yaremko BP, Chang A, Kundapur V, Mohan DS, Haas ML, Kim YB, Ferguson CL, Pugh SL, Kachnic LA and Bruner DW: Patient-reported toxicity during pelvic intensity-modulated radiation therapy: NRG Oncology-RTOG 1203. J Clin Oncol 36: 2538-2544, 2018. PMID: 29989857. DOI: $10.1200 / J C O .2017 .77 .4273$

9 Mell LK, Sirák I, Wei L, Tarnawski R, Mahantshetty U, Yashar CM, McHale MT, Xu R, Honerkamp-Smith G, Carmona R,
Wright M, Williamson CW, Kasaová L, Li N, Kry S, Michalski J, Bosch W, Straube W, Schwarz J, Lowenstein J, Jiang SB, Saenz CC, Plaxe S, Einck J, Khorprasert C, Koonings P, Harrison T, Shi $\mathrm{M}$ and Mundt AJ: Bone marrow-sparing intensity modulated radiation therapy with concurrent cisplatin for stage IB-IVA cervical cancer: An international multicenter phase II clinical trial (INTERTECC-2). Int J Radiat Oncol Biol Phys 97: 536-545, 2017. PMID: 28126303. DOI: 10.1016/ j.ijrobp.2016.11.027

10 Tamaki T, Hirai R, Igari M, Kumazaki Y, Noda S, Suzuki Y and Kato S: Dosimetric comparison of three-dimensional conformal radiotherapy versus volumetric-arc radiotherapy in cervical cancer treatment: Applying the central-shielding principle to modern technology. J Radiat Res 59: 639-648, 2018. PMID: 30053184. DOI: $10.1093 /$ jrr/rry054

11 Pötter R, Haie-Meder C, Van Limbergen E, Barillot I, De Brabandere M, Dimopoulos J, Dumas I, Erickson B, Lang S, Nulens A, Petrow P, Rownd J and Kirisits C: Recommendations from gynaecological (GYN) GEC ESTRO working group (II): Concepts and terms in 3D image-based treatment planning in cervix cancer brachytherapy-3D dose volume parameters and aspects of 3D image-based anatomy, radiation physics, radiobiology. Radiother Oncol 78: 67-77, 2006. PMID: 16403584. DOI: 10.1016/j.radonc.2005.11.014

12 Georg P, Kirisits C, Goldner G, Dörr W, Hammer J, Pötzi R, Berger D, Dimopoulos J, Georg D and Pötter R: Correlation of dose-volume parameters, endoscopic and clinical rectal side effects in cervix cancer patients treated with definitive radiotherapy including MRI-based brachytherapy. Radiother Oncol 91: 173-180, 2009. PMID: 19243846. DOI: 10.1016/j. radonc.2009.01.006

13 Mazeron R, Fokdal LU, Kirchheiner K, Georg P, Jastaniyah N, Šegedin B, Mahantshetty U, Hoskin P, Jürgenliemk-Schulz I, Kirisits C, Lindegaard JC and Dörr W: Dose-volume effect relationships for late rectal morbidity in patients treated with chemoradiation and MRI-guided adaptive brachytherapy for locally advanced cervical cancer: Results from the prospective multicenter EMBRACE study. Radiother Oncol 120: 412-419, 2016. PMID: 27396811. DOI: 10.1016/j.radonc.2016.06.006

14 Georg P, Pötter R, Georg D, Lang S, Dimopoulos JCA, Sturdza AE, Berger D, Kirisits C and Dörr W: Dose effect relationship for late side effects of the rectum and urinary bladder in magnetic resonance image-guided adaptive cervix cancer brachytherapy. Int J Radiat Oncol Biol Phys 82: 653-657, 2012. PMID: 21345618. DOI: 10.1016/j.ijrobp.2010.12.029

15 Kato S, Tran DN, Ohno T, Nakano T, Kiyohara H, Ohkubo Y and Kamada T: CT-based 3D dose-volume parameter of the rectum and late rectal complication in patients with cervical cancer treated with high-dose-rate intracavitary brachytherapy. J Radiat Res 51: 215-221, 2010. PMID: 20339256. DOI: 10.1269/ jrr.09118

16 Georg P, Lang S, Dimopoulos JC, Dörr W, Sturdza AE, Berger D, Georg D, Kirisits C and Pötter R: Dose-volume histogram parameters and late side effects in magnetic resonance imageguided adaptive cervical cancer brachytherapy. Int J Radiat Oncol Biol Phys 79: 356-362, 2011. PMID: 20385450. DOI: 10.1016/j.ijrobp.2009.11.002

17 Toita T, Ohno T, Kaneyasu Y, Kato T, Uno T, Hatano K, Norihisa Y, Kasamatsu T, Kodaira T, Yoshimura R, Ishikura S and Hiraoka M: A consensus-based guideline defining clinical 
target volume for primary disease in external beam radiotherapy for intact uterine cervical cancer. Jpn J Clin Oncol 41: 11191126, 2011. PMID: 21875938. DOI: 10.1093/jjco/hyr096

18 Toita T, Ohno T, Kaneyasu Y, Uno T, Yoshimura R, Kodaira T, Furutani K, Kasuya G, Ishikura S, Kamura T and Hiraoka M: A consensus-based guideline defining the clinical target volume for pelvic lymph nodes in external beam radiotherapy for uterine cervical cancer. Jpn J Clin Oncol 40: 456-463, 2010. PMID: 20133334. DOI: 10.1093/jjco/hyp191

19 Leicher B, Day E, Colonias A and Gayou O: Dosimetric comparison of IMRT rectal and anal canal plans generated using an anterior dose avoidance structure. Med Dosim 39: 272-275, 2014. PMID: 24913467. DOI: 10.1016/j.meddos.2014.05.001

20 Neill CJ: Dosimetric comparison of intensity-modulated solutions for intact prostate cancer. Med Dosim 39: 366-372, 2014. PMID: 25113813. DOI: 10.1016/j.meddos.2014.06.006

21 Tamaki T, Noda S, Ohno T, Kumazaki Y, Kato S and Nakano T: Dose-volume histogram analysis of composite EQD2 dose distributions using the central shielding technique in cervical cancer radiotherapy. Brachytherapy 15: 598-606, 2016. PMID: 27475482. DOI: 10.1016/j.brachy.2016.06.006

22 Tamaki T, Ohno T, Noda S, Kato S and Nakano T: Filling the gap in central shielding: Three-dimensional analysis of the EQD2 dose in radiotherapy for cervical cancer with the central shielding technique. J Radiat Res 56: 804-810, 2015. PMID: 26062811. DOI: $10.1093 / \mathrm{jrr} / \mathrm{rrv029}$

23 Macdonald DM, Lin LL, Biehl K, Mutic S, Nantz R and Grigsby PW: Combined intensity-modulated radiation therapy and brachytherapy in the treatment of cervical cancer. Int J Radiat Oncol Biol Phys 71: 618-624, 2008. PMID: 18407429. DOI: 10.1016/j.ijrobp.2008.02.014
24 Kidd EA, Siegel BA, Dehdashti F, Rader JS, Mutic S, Mutch DG, Powell MA and Grigsby PW: Clinical outcomes of definitive intensity-modulated radiation therapy with fluorodeoxyglucose-positron emission tomography simulation in patients with locally advanced cervical cancer. Int J Radiat Oncol Biol Phys 77: 1085-1091, 2010. PMID: 19880262. DOI: 10.1016/j.ijrobp.2009.06.041

25 Perez CA and Kavanagh BD: Uterine cervix. In: Principles and Practice of Radiation Oncology. Fourth Edition. Perez CA, Brady LW, Halperin EC and Schmidt-Ullrich RK (eds.). Philadelphia, PA, USA, Lippincott Williams \& Wilkins, pp. 1800-1915, 2003.

26 Cheng JY, Huang EY, Hsu SN and Wang CJ: Simultaneous integrated boost (SIB) of the parametrium and cervix in radiotherapy for uterine cervical carcinoma: A dosimetric study using a new alternative approach. Br J Radiol 89: 1-10, 2016. PMID: 27706947. DOI: 10.1259/bjr.20160526
Received August 28, 2020

Revised September 10, 2020 Accepted September 14, 2020 